\title{
Use and effectiveness of dapagliflozin in patients with type 2 diabetes mellitus: a multicenter retrospective study in Taiwan
}

\author{
Jung-Fu Chen ${ }^{1,2}$, Yun-Shing Peng ${ }^{3}$, Chung-Sen Chen ${ }^{4}$, Chin-Hsiao Tseng ${ }^{5}$, Pei-Chi Chen ${ }^{6}$, Ting-I Lee ${ }^{7,8}$, Yung- \\ Chuan Lu ${ }^{9,10}$, Yi-Sun Yang ${ }^{11}$, Ching-Ling Lin ${ }^{8,12}$, Yi-Jen Hung ${ }^{13}$, Szu-Ta Chen ${ }^{14}$, Chieh-Hsiang Lu ${ }^{9,15,16}$, Chwen-Yi

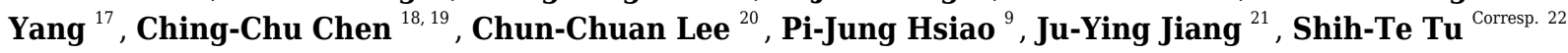 \\ 1 Division of Endocrinology and Metabolism, Department of Internal Medicine, Kaohsiung Chang Gung Memorial Hospital, Kaohsiung, Taiwan \\ 2 College of Medicine, Chang Gung University, Taoyuan, Taiwan \\ 3 Division of Endocrinology and Metabolism, Department of Internal Medicine, Chiayi Chang Gung Memorial Hospital, Chiayi, Taiwan \\ 4 Division of Endocrinology and Metabolism, Department of Internal Medicine, Taipei City Hospital Zhongxiao Branch, Taipei, Taiwan \\ 5 Division of Endocrinology and Metabolism, Department of Internal Medicine, National Taiwan University Hospital, Taipei, Taiwan \\ 6 \\ 6 Division of Endocrinology and Metabolism, Department of Internal Medicine, Shin Kong Wu Ho-Su Memorial Hospital, Taipei, Taiwan \\ 7 Division of Endocrinology and Metabolism, Department of Internal Medicine, Wan Fang Hospital, Taipei Medical University, Taipei, Taiwan \\ 8 \\ Department of General Medicine, School of Medicine, College of Medicine, Taipei Medical University, Taipei, Taiwan \\ 9 Division of Endocrinology and Metabolism, Department of Internal Medicine, E-Da Hospital, Kaohsiung, Taiwan \\ 10 School of Medicine, College of Medicine, I-Shou University, Kaohsiung, Taiwan \\ 11 Division of Endocrinology and Metabolism, Department of Internal Medicine, Chung Shan Medical University Hospital, Taichung, Taiwan \\ 12 Division of Endocrinology and Metabolism, Department of Internal Medicine, Cathay General Hospital, Taipei, Taiwan \\ 13 Division of Endocrinology and Metabolism, Department of Internal Medicine, Tri-Service General Hospital Songshan Branch, Taipei, Taiwan \\ 14 Division of Endocrinology and Metabolism, Department of Internal Medicine, Linkou Chang Gung Memorial Hospital, Taoyuan, Taiwan \\ 15 Division of Endocrinology and Metabolism, Department of Internal Medicine, Chia-Yi Christian Hospital, Chia-Yi, Taiwan \\ 16 Lutheran Medical Foundation, Kaohsiung Christian Hospital, Kaohsiung, Taiwan \\ 17 Division of Endocrinology and Metabolism, Department of Internal Medicine, Chi Mei Medical Center, Tainan, Taiwan \\ 18 Division of Endocrinology and Metabolism, Department of Medicine, China Medical University Hospital, Taichung, Taiwan \\ 19 School of Chinese Medicine, China Medical University, Taichung, Taiwan \\ 20 Division of Endocrinology and Metabolism, Department of Internal Medicine, Mackay Memorial Hospital, Taipei, Taiwan \\ 21 Division of Endocrinology and Metabolism, Department of Internal Medicine, Far Eastern Memorial Hospital, New Taipei City, Taiwan \\ 22 Division of Endocrinology and Metabolism, Department of Internal Medicine, Changhua Christian Hospital, Changhua, Taiwan \\ Corresponding Author: Shih-Te Tu \\ Email address: 10836@cch.org.tw
}

Aims/Introduction. To investigate the clinical outcomes of patients with type 2 diabetes mellitus (T2DM) who initiated dapagliflozin in real-world practice in Taiwan.

Materials and Methods. In this multicenter retrospective study, adult patients with T2DM who initiated dapagliflozin after May $1^{\text {st }} 2016$ either as add-on or switch therapy were included. Changes in clinical and laboratory parameters were evaluated at 3 and 6 months. Baseline factors associated with dapagliflozin response in glycated hemoglobin ( $\mathrm{HbAlc}$ ) were analyzed by univariate and multivariate logistic regression.

Results. A total of 1960 patients were eligible. At 6 months, significant changes were observed: HbAlc by $-0.73 \%(95 \%$ confidence interval $[\mathrm{Cl}]-0.80,-0.67)$, body weight was $-1.61 \mathrm{~kg}(95 \% \mathrm{Cl}-1.79,-1.42)$,

Peer) reviewing PDF | (2020:02:45716:2:0:CHECK 12 Aug 2020) 
and systolic/diastolic blood pressure by -3.6/-1.4 mmHg. Add-on dapagliflozin showed significantly greater $\mathrm{HbAlc}$ reduction $(-0.82 \%)$ than switched therapy $(-0.66 \%)(p=0.0023)$. The proportion of patients achieving $\mathrm{HbAlc}<7 \%$ target increased from $6 \%$ at baseline to $19 \%$ at Month 6 . Almost $80 \%$ of patients experienced at least $1 \%$ reduction in $\mathrm{HbAlC}$, and $65 \%$ of patients showed both weight loss and reduction in $\mathrm{HbAlc}$. Around $37 \%$ of patients had at least 3\% weight loss. Multivariate logistic regression analysis indicated patients with higher baseline $\mathrm{HbAlc}$ and those who initiated dapagliflozin as add-on therapy were associated with a greater reduction in HbAlc.

Conclusions. In this real-world study with the highest patient number of Chinese population to date, the use of dapagliflozin was associated with significant improvement in glycemic control, body weight, and blood pressure in patients with T2DM. Initiating dapagliflozin as add-on therapy showed better glycemic control than as switch therapy. 
1 Use and effectiveness of dapagliflozin in patients with

2 type 2 diabetes mellitus: a multicenter retrospective

3 study in Taiwan

4

5

6 7

Jung-Fu Chen ${ }^{1,2}$, Yun-Shing Peng ${ }^{3}$, Chung-Sen Chen ${ }^{4}$, Chin-Hsiao Tseng ${ }^{5}$, Pei-Chi Chen ${ }^{6}$, TingI Lee $^{7,8}$, Y ung-Chuan Lu ${ }^{9,10}$, Yi-Sun Yang ${ }^{11}$, Ching-Ling Lin ${ }^{12}$, Yi-Jen Hung ${ }^{13}$, Szu-Ta Chen ${ }^{14}$, Chieh-Hsiang Lu ${ }^{15-17}$, Chwen-Yi Yang ${ }^{18}$, Ching-Chu Chen ${ }^{19,20}$, Chun-Chuan Lee ${ }^{21}$, Pi-Jung Hsiao $^{17}$, Ju-Ying Jiang ${ }^{22}$, Shih-Te Tu ${ }^{23}$

1. Division of Endocrinology and Metabolism, Department of Internal Medicine, Kaohsiung Chang Gung Memorial Hospital, Kaohsiung, Taiwan

2. College of Medicine, Chang Gung University, Kaohsiung, Taiwan

3. Division of Endocrinology and Metabolism, Department of Internal Medicine, Chiayi Chang Gung Memorial Hospital, Taiwan

4. Division of Endocrinology and Metabolism, Department of Internal Medicine, Taipei City Hospital Zhongxiao Branch, Taipei, Taiwan

5. Division of Endocrinology and Metabolism, Department of Internal Medicine, National Taiwan University Hospital, Taipei, Taiwan

6. Division of Endocrinology and Metabolism, Department of Internal Medicine, Shin Kong $\mathrm{Wu}$ Ho-Su Memorial Hospital, Taipei, Taiwan

7. Division of Endocrinology and Metabolism, Department of Internal Medicine, Wan Fang Hospital, Taipei Medical University, Taipei, Taiwan

8. Taipei Medical University, Taipei, Taiwan

9. Division of Endocrinology and Metabolism, Department of Internal Medicine, E-Da Hospital, Kaohsiung, Taiwan

10. School of Medicine, College of Medicine, I-Shou University, Kaohsiung, Taiwan

11. Division of Endocrinology and Metabolism, Department of Internal Medicine, Chung Shan Medical University Hospital, Taichung, Taiwan

12. Division of Endocrinology and Metabolism, Department of Internal Medicine, Cathay General Hospital, Taipei, Taiwan

13. Division of Endocrinology and Metabolism, Department of Internal Medicine, Tri-Service General Hospital Songshan Branch, Taipei, Taiwan

14. Division of Endocrinology and Metabolism, Department of Internal Medicine, Linkou Chang Gung Memorial Hospital, Taoyuan, Taiwan

15. Division of Endocrinology and Metabolism, Department of internal medicine, Chia-Yi Christian Hospital, Chia-Yi, Taiwan 
39

40

41

42

43

44

45

46

47

48

49

50

51

52

53

54

55

56

57

58

59

60

61

62

63

64

65

66

67

68

69

70

16. Lutheran Medical Foundation, Kaohsiung Christian Hospital, Kaohsiung, Taiwan

17. Kaohsiung Medical University, Kaohsiung, Taiwan

18. Division of Endocrinology and Metabolism, Department of Internal Medicine, Chi Mei Medical Center, Tainan, Taiwan

19. Division of Endocrinology and Metabolism, Department of Internal Medicine, China Medical University Hospital, Taichung, Taiwan

20. School of Chinese Medicine, China Medical University, Taichung, Taiwan

21. Division of Endocrinology and Metabolism, Department of Internal Medicine, Mackay Memorial Hospital, Taipei, Taiwan

22. Division of Endocrinology and Metabolism, Department of Internal Medicine, Far Eastern Memorial Hospital, New Taipei City, Taiwan

23. Division of Endocrinology and Metabolism, Department of Internal Medicine, Changhua Christian Hospital, Changhua, Taiwan

Corresponding Author:

Shih-Te Tu MD 23

135 Nanhsiao Street, Changhua City 50006, TAIWAN

Email address: 10836@cch.org.tw

\section{Abstract}

Aims/Introduction. To investigate the clinical outcomes of patients with type 2 diabetes

mellitus (T2DM) who initiated dapagliflozin in real-world practice in Taiwan.

Materials and Methods. In this multicenter retrospective study, adult patients with T2DM who initiated dapagliflozin after May $1^{\text {st }} 2016$ either as add-on or switch therapy were included.

Changes in clinical and laboratory parameters were evaluated at 3 and 6 months. Baseline factors associated with dapagliflozin response in glycated hemoglobin (HbA1c) were analyzed by univariate and multivariate logistic regression.

Results. A total of 1960 patients were eligible. At 6 months, significant changes were observed: HbA1c by $-0.73 \%$ (95\% confidence interval [CI] $-0.80,-0.67)$, body weight was $-1.61 \mathrm{~kg}(95 \%$ CI -1.79, -1.42), and systolic/diastolic blood pressure by -3.6/-1.4 mmHg. Add-on dapagliflozin showed significantly greater $\mathrm{HbA} 1 \mathrm{c}$ reduction $(-0.82 \%)$ than switched therapy $(-0.66 \%)$ $(p=0.002)$. The proportion of patients achieving HbA1c $<7 \%$ target increased from $6 \%$ at 
71 baseline to $19 \%$ at Month 6 . Almost $80 \%$ of patients experienced at least $1 \%$ reduction in

$72 \mathrm{HbA} 1 \mathrm{c}$, and $65 \%$ of patients showed both weight loss and reduction in $\mathrm{HbA} 1 \mathrm{c}$. Around $37 \%$ of

73 patients had at least 3\% weight loss. Multivariate logistic regression analysis indicated patients

74 with higher baseline HbAlc and those who initiated dapagliflozin as add-on therapy were

75 associated with a greater reduction in $\mathrm{HbAlc}$.

76 Conclusions. In this real-world study with the highest patient number of Chinese population to

77 date, the use of dapagliflozin was associated with significant improvement in glycemic control,

78 body weight, and blood pressure in patients with T2DM. Initiating dapagliflozin as add-on

79 therapy showed better glycemic control than as switch therapy.

80

\section{Introduction}

82

83

84

85

86

87

88

89

90

91

92

93

94

95

96

97

98

99

100

101

102

103

Type 2 diabetes mellitus (T2DM) is a chronic metabolic disease affecting populations worldwide, and it has become an important public health challenge among Asian countries, especially in ethnic Chinese populations.(Cho et al. 2018; Lim \& Chan 2019; Lim et al. 2017; Nanditha et al. 2016) According to the 2019 IDF Diabetes Atlas and a nationwide database analysis in Taiwan, the prevalence of diabetes increase from $4.31 \%$ in 2000 to $6.6 \%$ in 2019 for adults aged $20-79$ years, resulting in a more than $70 \%$ increase in the total diabetic population (1.23 million in 2019).(International Diabetes Federation 2019; Jiang et al. 2012) Currently, most guidelines recommend pharmacologic therapy based on evaluating glycated hemoglobin (HbA1c) levels for glycemic control. When the glycemic target is not achieved by lifestyle management and metformin, a second agent may be initiated, considering medication profiles and patient-related factors.(American Diabetes Association 2019; Diabetes Association Of The Republic Of China Taiwan 2019; Garber et al. 2018; McGuire et al. 2016)

Sodium-glucose cotransporter-2 (SGLT2) inhibitors are a newer class of oral antidiabetic drugs (OADs) that inhibit glucose reabsorption at the early segments of the proximal convoluted tubule, thereby promoting glucosuria independently of insulin action.(Hasan et al. 2014) These agents improve glycemic control without increasing the risk of hypoglycemia, and have pleiotropic effects such as weight loss and reduction in blood pressure (BP). Combining SGLT2 inhibitors with metformin has been demonstrated to have additive effect compared to metformin alone in HbA1c and body weight reduction.(Molugulu et al. 2017) Given that T2DM has been known to have a higher risk of cardiovascular events, significant attention has been paid to the benefit of SGLT2 inhibitors on cardiovascular outcomes in T2DM patients with or without pre-existing cardiovascular disease.(Scholtes et al. 2019) 
104

105

106

107

108

109

110

111

112

113

114

115

116

117

118

119

120

121

122

123

124

125

126

127

128

129

130

131

132

133

134

135

136

137

138

139

140

141

142

Dapagliflozin, a member of the SGLT2 inhibitor class, has been shown in randomized controlled trials (RCTs) to improve glycemic control both as monotherapy(Bailey et al. 2015) and as add-on to other antidiabetic drugs,(Nauck et al. 2014; Sun et al. 2014) along with wellestablished safety profile in a pooled analysis of phase IIb/III trials.(Jabbour et al. 2018) Complimentary to RCTs, observational studies can provide real-world data reflecting clinical practice patterns and outcomes not collected in RCTs.(Garrison et al. 2007)

The real-world evidence on dapagliflozin has been reported in North America(Brown et al. 2017; Huang et al. 2018b) and Europe,(Fadini et al. 2018; Mirabelli et al. 2019; Scheerer et al. 2016; Scorsone et al. 2018; Wilding et al. 2017) whereas data are limited in Asia, especially for ethnic Chinese populations.(Han et al. 2018; Tobita et al. 2017; Viswanathan \& Singh 2019) Dapagliflozin has been reimbursed by the National Health Insurance (NHI) in Taiwan since May of 2016. The purpose of the LEAD (Learning from RWE: A Multicenter retrospective study of Dapagliflozin in Taiwan) study was to retrospectively investigate the clinical outcomes for patients with T2DM initiating dapagliflozin under real-world setting in Taiwan.

\section{Materials \& Methods}

Data source and study population

This was a multicenter retrospective observational study (ClinicalTrials.gov identifier NCT03084965) enrolling patients with T2DM exposed to dapagliflozin after dapagliflozin reimbursement in Taiwan since May 2016. Medical information was extracted manually by chart review and recorded in a standardized case report form after obtaining written informed consent from the patients. The final protocol of this study, including the final version of the subject informed consent form, had been approved by the Ethics Committee/Institutional Review Board (IRB)/Independent Ethics Committee (IEC) of each sites (Institutional Review Board Changhua Christian Hospital, Research Ethics Committee China Medical University \& Hospital, The Institutional Review Board Chung Shan Medical University Hospital, Institutional Review Board Chia-Yi Hospital, Taipei City Hospital Research Ethics Committee, Chang Gung Medical Foundation Institutional Review Board, Kaohsiung Medical University Chung-Ho Memorial Hospital, Institutional Review Board Chi Mei Medical Center, Institutional Review Board of the E-DA Hospital, The Institutional Review Board of Shin Kong Wu Ho-Su Memorial Hospital, Institutional Review Board, Tri-Service General Hospital, Research Ethics Review Committee Far Eastern Memorial Hospital, Institutional Review Board of the Cathay General Hospital, TMUJoint Institutional Review Board, National Taiwan University Hospital Research Ethics Committee, Institutional Review Board MacKay Memorial Hospital).

Patients were eligible for inclusion if they (1) were diagnosed with T2DM and aged $\geq 20$ years old, (2) initiated $5 \mathrm{mg}$ or $10 \mathrm{mg}$ of dapagliflozin after May $1^{\text {st }} 2016$, either as add-on therapy to existing $\mathrm{OAD}(\mathrm{s})$ with or without insulin, or as switch therapy from another OAD, and (3) completed follow-up of at least 6 months regardless of continuation on dapagliflozin therapy. Data were excluded if they (1) received other SGLT2 inhibitors prior to the initiation of dapagliflozin, 
143 (2) had a diagnosis of type 1 diabetes, or (3) were included in other clinical trials concurrently

144 during the retrospective data collection period.

145

146

147

148

149

150

151

152

153

154

155

156

157

158

159

160

161

162

163

164

165

166

167

168

169

170

171

172

173

174

175

176

177

178

179

180

181

182

\section{Assessment and outcome measures}

Patient baseline demographics and clinical characteristics were recorded at the time of dapagliflozin initiation. Reasons for starting/switching to dapagliflozin, rate and reasons of dapagliflozin discontinuation were collected. Measurements of HbAlc, body weight, BP, fasting plasma glucose (FPG), and lipid profile (total cholesterol, high-density lipoprotein cholesterol [HDL-C], low-density lipoprotein cholesterol [LDL-C], and triglycerides) were obtained at baseline, 3 and 6 months to assess for changes. Further subgroup analyses were performed to assess changes in HbA1c by baseline HbA1c, manner of dapagliflozin initiation (as add-on or switch), BMI, age, and dosage of dapagliflozin. Changes in body weight were also analyzed by subgroups of baseline BMI, HbA1c, age, and dosage of dapagliflozin. Patients who have one or more times record using $5 \mathrm{mg}$ dapagliflozin during the follow up were defined as $5 \mathrm{mg}$ group. The proportion of patients within each glycemic level (HbA1c $<7 \%, 7-8 \%, 8-9 \%$, and $>9 \%$ ) was evaluated at baseline and 6 months.

\section{Statistical analysis}

Descriptive statistics were provided for all variables. Continuous variables were presented with mean \pm standard deviation, and numbers or percentages were used for categorical variables. Changes from baseline of the variables were analyzed using evaluable data at the respective time points. Mean changes of each variable from baseline to Month 3, baseline to Month 6, and between 3 and 6 months were evaluated by paired $t$-test and Wilcoxon signed-rank test in the overall cohort and subgroups. Differences in HbA1c reduction between dapagliflozin add-on and switch groups at Month 3 and Month 6 were analyzed by Wilcoxon rank-sum test. For changes in HbA1c and body weight among other subgroups at Month 3 and Month 6, the Kruskal-Wallis test was used. Patient distributions of changes in HbAlc and body weight at Month 6 were displayed by scatter plots for total, add-on, and switch groups. Baseline factors associated with dapagliflozin response in $\mathrm{HbA} 1 \mathrm{c}$ were examined by univariate and multivariate logistic regression analyses. The cutoff for dapagliflozin responders was determined by using the median of change in HbAlc in patients with evaluable data at Month 6 . Regarding the missing data, patients without the respective data were excluded in each analysis. For instance, when analyzing change from baseline to Month 3 , only patients with both baseline and Month 3 data were included. Statistical significance was set at $p$ value $<0.05$. All statistical analyses were conducted using SAS version 9.3.

\section{Results}

\section{Baseline characteristics}

A total of 1960 patients were eligible, with a mean age of $57.8 \pm 11.5$ years, and $52 \%$ were male. The baseline clinical and laboratory characteristics are shown in Table 1. The mean HbA1c was $8.8 \pm 1.4 \%$, and body weight was $75.2 \pm 15.8 \mathrm{~kg}$. Baseline systolic blood pressure (SBP) and 
183

184

185

186

187

188

189

190

191

192

193

194

195

196

197

198

199

200

201

202

203

204

205

206

207

208

209

210

211

212

213

214

215

216

217

218

219

220

221

222

diastolic blood pressure (DBP) was $136.6 \pm 17.6$ and $77.8 \pm 11.3 \mathrm{mmHg}$, respectively. Metformin was the most prescribed antihyperglycemic agent (92.4\%), followed by sulfonylurea $(70.7 \%)$ and dipeptidyl peptidase-4 (DPP4) inhibitor (53.5\%). Over half of the patients were on either dual $(38.2 \%)$ or triple $(36.2 \%)$ therapy prior to initiating dapagliflozin. A large proportion of patients $(78.9 \%)$ initiated dapagliflozin at the higher dose $(10 \mathrm{mg})$. Regarding the manner of initiation, more than half $(53.8 \%)$ of the patients were switched to dapagliflozin, while the others $(46.2 \%)$ started dapagliflozin as add-on therapy. Main reasons for starting dapagliflozin were for its HbA1c lowering efficacy $(81.2 \%)$, followed by less concern for weight gain $(26.8 \%)$ and lower risk of hypoglycemia $(11.4 \%)$.

\section{Effectiveness of dapagliflozin on clinical and laboratory parameters}

Table 2 shows changes in clinical and laboratory parameters after dapagliflozin initiation at 3 and 6 months. Compared with baseline, statistically significant reductions in HbAlc were observed both at Month $3(-0.68 \%$; 95\% confidence interval [CI] $-0.74,-0.62, p<0.001)$ and at Month $6(-$ $0.73 \% ; 95 \%$ CI $-0.80,-0.67, p<0.001)$. Considering patients with evaluable HbA1c data at baseline and Month 6, the proportion of patients achieving the glycemic target (HbA1c $<7 \%)$ was $6 \%$ at baseline, and it increased to $19 \%$ by Month 6 after initiating dapagliflozin. Moreover, the proportion of patients who were poorly controlled (HbAlc $>9 \%$ ) decreased from $34.7 \%$ at baseline to $15.9 \%$ at Month 6 (Fig S1A). Improvements in FPG (-28.3 mg/dL), BMI (-0.60), body weight $(-1.61 \mathrm{~kg})$, and SBP/DBP $(-3.6 /-1.4 \mathrm{mmHg})$ at Month 6 were also significant (all $p<0.001$, Table 2). Aside from BP reduction that plateaued at Month 3, small but significant improvements were observed in HbA1c, FPG, BMI, and body weight from Month 3 to Month 6. Small changes in lipid profiles were also noted (Table 2).

\section{Subgroup analyses: HbAlc and body weight}

We performed analyses to examine the effects of dapagliflozin treatment on HbAlc and body weight among different subgroups. For $\mathrm{HbA1c}$, a statistically significant trend of greater reduction was observed in patients with higher baseline HbA1c from baseline to 3 and 6 months (Fig. 1A). In patients with baseline HbA1c between $8-9 \%$ and those $>10 \%$, a significant greater HbAlc reduction was found at Month 6 compared with Month3. Patients who received dapagliflozin as add-on therapy had a significantly greater reduction in HbAlc $(-0.82 \%)$ than those who were switched from one antihyperglycemic agent to dapagliflozin at Month $6(-0.66 \%, p=0.002$, Fig. 1B). Considering patients with evaluable HbAlc data at baseline and Month 6 , the proportion of patients achieving the glycemic target $(\mathrm{HbA} 1 \mathrm{c}<7 \%)$ were $5 \%$ and $6 \%$ for dapagliflozin add-on and switch groups at baseline, and they subsequently increased to $23 \%$ and $15 \%$ by Month 6 , respectively (Fig. S1B). When stratified by baseline BMI, no significant difference in HbA1c reduction was found across subgroups (Fig. S2A). Similarly, no difference among age subgroups was found for HbA1c reduction (Fig. S2B). When stratified by dosage of dapagliflozin, patients receive $10 \mathrm{mg}$ dapagliflozin had significantly greater reduction in $\mathrm{HbAlc}(-0.74 \%)$ than those who receive $5 \mathrm{mg}$ at month $6(-0.67 \%, \mathrm{p}<0.001$, Fig. S2C). 
223

224

225

226

227

228

229

230

231

232

233

234

235

236

237

238

239

240

241

242

243

244

245

246

247

248

249

250

251

252

253

254

255

256

257

258

259

260

261

262

For body weight, treatment with dapagliflozin showed a statistically significant trend of greater weight loss with increasing baseline BMI from baseline to 3 and 6 months (Fig. 2). Among patients with evaluable data at both Month 3 and Month 6, further weight reduction was significant at Month 6 compared with Month 3 across all BMI categories, except for those with baseline BMI $\geq 35$. When stratified by baseline HbAlc, significantly greater weight reductions were found in patients with lower baseline HbA1c throughout the study, despite similar baseline body weight among HbA1c categories (Fig. S3A). There was no difference in weight reduction across age subgroups at Month 3, but a significant difference was found at Month 6 (Fig. S3B). Comparing data between 3 and 6 months, significant reductions in weight were found in groups aged 40-65 and 65-75 years. When stratified by dosage of dapagliflozin, patients receive $10 \mathrm{mg}$ dapagliflozin had significantly greater reduction in body weight $(-1.65 \mathrm{~kg})$ than those who receive $5 \mathrm{mg}$ at month $6(-1.43 \mathrm{~kg}, \mathrm{p}=0.011$, Fig. S3C).

In addition, patients were stratified into four groups according to the number of antidiabetic therapies at baseline: monotherapy, dual therapy, triple therapy, and $>3$ drugs combination. Across these subgroups, no significant differences were observed in both $\mathrm{HbA1c}$ and body weight reductions (Fig. S2D; Fig. S3D).

\section{Relationship between changes in HbAlc and body weight}

Patient distributions of changes in HbAlc and body weight after initiating dapagliflozin for 6 months were presented in scatter plots (Fig. 3). Of the 1094 patients with evaluable data, 79.9\% and $77.6 \%$ of them experienced reductions in HbA1c and body weight, respectively. Moreover, $64.0 \%$ of patients showed simultaneous reductions in both outcomes (Fig. 3A). For patients in the add-on ( $\mathrm{n}=529)$ and switch ( $\mathrm{n}=565)$ groups, $69.2 \%$ and $59.4 \%$ had a reduction in both HbA1c and body weight, respectively (Fig. 3B and 3C). In terms of clinical meaningful change, $74.4 \%$ and $74.2 \%$ of patients had at least $0.5 \%$ and $1 \%$ reduction in HbAlc for 6 months, respectively. $37.1 \%$ of patients had at least 3\% weight loss for 6 months. The baseline characteristics of at least $1 \%$ reduction in $\mathrm{HbA1c}, 3 \%$ weight loss and both were show in the supplement table 1-3, respectively.

\section{Baseline factors associated with dapagliflozin response in $\mathrm{HbAlc}$}

Baseline factors associated with dapagliflozin response in HbA1c at Month 6 were shown in Table 3. Responders and non-responders were determined by using the median of change in HbA1c ($0.60 \%$; min, $\max [-6.6,3.2])$ as the cutoff.

Univariate logistic regression analysis indicated that higher baseline HbAlc, FPG, add-on dapagliflozin, use of insulin were significantly associated with dapagliflozin response in HbA1c reduction, while being on dual therapy at the time of dapagliflozin initiation was significantly associated with less $\mathrm{HbA1c}$ reduction. In multivariate logistic regression analysis, significant associations with dapagliflozin response in $\mathrm{HbAlc}$ reduction were found in patients with higher baseline HbA1c (odds ratio [OR] 2.10; 95\% CI 1.79-2.47, $p<0.001$ ), and those who received dapagliflozin as add-on therapy (OR 1.60; 95\% CI 1.19-2.14, $p=0.002$ ). 
263

264

265

266

267

268

269

270

271

272

273

274

275

276

277

278

279

280

281

282

283

284

285

286

287

288

289

290

291

292

293

294

295

296

297

298

299

300

301

302

\section{Discontinue rate and reasons for discontinuation}

Among eligible patients, the total number of discontinuation at Month 3 and Month 6 were 153 $(7.8 \%)$ and $247(12.6 \%)$, respectively. The main reasons for the discontinuation were inadequate HbAlc control $(n=83 ; 4.23 \%)$, intolerance $(n=46 ; 2.35 \%)$, or poor compliance to the current regimens $(\mathrm{n}=9 ; 0.46 \%)$. The most commonly reported reasons for intolerance were genital or urinary tract infections $(n=15)$, frequency of urination $(n=8)$, and vaginal itching $(n=5)$.

\section{Discussion}

This multicenter retrospective study presented the first nationwide real-world evidence for dapagliflozin in Taiwan. In this cohort of 1960 Taiwanese patients with T2DM, initiating dapagliflozin was associated with significant improvements in glycemic control, body weight, and BP. Overall, the changes from baseline were $-0.73 \%$ for $\mathrm{HbA} 1 \mathrm{c},-28.3 \mathrm{mg} / \mathrm{dL}$ for FPG, $-1.61 \mathrm{~kg}$ ($2.14 \%$ ) for body weight, and $-3.6 /-1.4 \mathrm{mmHg}$ for SBP/DBP at 6 months. These clinical benefits of dapagliflozin are comparable to the efficacy data from meta-analyses of RCTs.(Sun et al. 2014; Zhang et al. 2014) In addition, small changes in lipids were noted, including an increase in total cholesterol, LDL-C, HDL-C, and a decrease in triglycerides.

Several observation studies assessing the effectiveness of dapagliflozin have been published recently.(Brown et al. 2017; Fadini et al. 2018; Han et al. 2018; Huang et al. 2018b; Mirabelli et al. 2019; Scheerer et al. 2016; Viswanathan \& Singh 2019; Wilding et al. 2017) Despite differences in ethnicity, location, and other clinical factors, the baseline HbAlc among studies were similar to our data, ranging $8.5 \%-9.5 \%$, and many patients were already on two or three OADs, some were even using insulin. The effects of dapagliflozin on glycemic control, body weight, and BP were consistent across these studies: reduction in $\mathrm{HbA1c}$ ranged from $0.7 \%-1.5 \%$ over 6 to 12 months of observation, reduction in body weight by percentage ranged $1.75 \%-3.83 \%$, and reduction for SBP and DBP were of 2.3-3.8 $\mathrm{mmHg}$ and 1.1-2.0 $\mathrm{mmHg}$, respectively. In our study, these effects were significant 3 months after initiating dapagliflozin, and aside from the BPlowering effect that plateaued at Month 3, we found small but significant improvements in other key metabolic parameters from Month 3 to Month 6 . Also, 65\% of patients showed simultaneous reductions in both $\mathrm{HbA1c}$ and body weight, comparable to the previous reports.(Fadini et al. 2018; Han et al. 2018; Scheerer et al. 2016) These data suggested that dapagliflozin is effective in realworld practice for patients with inadequately controlled T2DM.

The baseline characteristics in our cohort, such as mean age, sex ratio, and HbAlc were similar to the South-East Asia cohort in the recent global DISCOVER study.(Gomes et al. 2019) Although diabetic patients in South-East Asia (and Western Pacific region) have lower BMI than those in Western countries, the current Taiwanese study had slightly higher BMI compared with the South-East Asia data in the DISCOVER study (28.3 vs. 27.3). To explore and identify whether some baseline factors may be associated with better treatment response to dapagliflozin, subgroup and logistic regression analyses were performed. We observed a significant trend of greater $\mathrm{HbAlc}$ reduction in patients with higher baseline $\mathrm{HbAlc}$, and the association was further indicated in multivariate logistic regression analysis. This association is consistent with findings from the 
303 literature. In general, studies suggest that patients with higher baseline HbAlc experienced a

304

305

306

307

308

309

310

311

312

313

314

315

316

317

318

319

320

321

322

323

324

325

326

327

328

329

330

331

332

333

334

335

336

337

338

339

340

341

342

greater reduction in HbA1c with dapagliflozin treatment,(Brown et al. 2017; Han et al. 2018; Hong et al. 2019; Scheerer et al. 2016) and one study indicates that a lower baseline HbA1c was associated with the achievement of HbAlc goal $(<7 \%)$.(Wilding et al. 2017) Other patient characteristics that may be associated with better dapagliflozin response were male,(Brown et al. 2017) younger age $(<45$ years old),(Wilding et al. 2017) shorter disease duration $(<2-4$ years),(Brown et al. 2017; Han et al. 2018; Wilding et al. 2017) and non-insulin use(Brown et al. 2017; Hong et al. 2019); however, some of these associations were not identified and other data were not available in the current study. On the other hand, baseline body weight or BMI did not influence the magnitude of reduction in $\mathrm{HbAlc}$, which is similar to previous studies.(Han et al. 2018; Scheerer et al. 2016; Wilding et al. 2017)

Regarding the effects of dapagliflozin by manners of initiation, we found patients who initiated dapagliflozin as add-on therapy had a significantly greater reduction in HbAlc $(-0.82 \%)$ than those who were switched $(-0.66 \%)$ from other OADs. Two recent studies also showed treatment with SGLT2 inhibitors (i.e., dapagliflozin or empagliflozin) as add-on therapy had a greater glucose-lowering effect than as switch therapy.(Han et al. 2018; Hong et al. 2019) Notably, we found DPP4 inhibitors accounted for the majority (69.5\%) of the switched agents (data not shown), a finding which is likely due to the fact that Taiwan's NHI only covers either a DPP4 inhibitor or an SGLT2 inhibitor. A single-center retrospective study in Taiwan recently reported that patients who switched from a DPP4 inhibitor to an SGLT2 inhibitor (i.e., empagliflozin) for 6 months had significant reductions in HbAlc and body weight, whereas those who remained on a DPP4 inhibitor did not experience significant changes.(Huang et al. 2018a) Moreover, data from long-term clinical trials demonstrated sustained efficacy and tolerability of dapagliflozin as addon therapy.(Bailey et al. 2013; Del Prato et al. 2015; Leiter et al. 2016; Matthaei et al. 2015) Taken together, switching to an SGLT2 inhibitor from other OADs such as DPP4 inhibitors may be a suitable option for antidiabetic treatment, and add-on SGLT2 inhibition could provide better clinical benefit than switch therapy in patients already taking other OADs without adequate glycemic control.(Hong et al. 2019; Huang et al. 2018a)

In our study, we observed a significant trend of greater weight loss with increasing baseline BMI. This trend remained when data were calculated by the percentage of weight reduction, with about $1 \%$ weight reduction in the BMI $<24$ category and over $2 \%$ in those with $\mathrm{BMI} \geq 30$ (data not shown). Besides, we also observed a significant weight loss in patients received $10 \mathrm{mg}$ dapagliflozin compared with $5 \mathrm{mg}$. This result is similar with a previous study that have demonstrate a dose-dependent reduction in body weight with dapagliflozin in Chinese patients under placebo-controlled trial conditions.(Cai et al. 2018) Loss of fat mass has been known to account for approximately $70 \%$ of total weight loss observed with dapagliflozin treatment.(Bolinder et al. 2012) These findings have significant clinical implications in Asians, as visceral adiposity is known to be higher in Asians than Caucasians at a given BMI, contributing to insulin resistance that leads to cardiovascular and renal complication.(Lim et al. 2017; Ma \& Chan 2013) Given its effectiveness on HbA1c, FPG, body weight, and BP, dapagliflozin could serve as

Peer) reviewing PDF | (2020:02:45716:2:0:CHECK 12 Aug 2020) 
343 a promising OAD for Asian population to achieve better glycemic control and reduce future risk 344 of cardiovascular diseases.

345 Since several cardiovascular outcome trials of SGLT2 inhibitors have been published, their 346 effects on cardiorenal protection have come under the spotlight.(Scholtes et al. 2019) Both large347 scale RCT and real-world studies (e.g. CVD-REAL study) indicate that dapagliflozin lowers 348 hospitalization for heart failure and cardiovascular mortality in T2DM patients with existing or

349

350

351

352

353

354

355

356

357

358

359

360

361

362

363

364

365

366

367

368

369

370

371

372

373

374

375

376

377

378

379

380

381 risk of cardiovascular disease.(Cavender et al. 2018; Norhammar et al. 2019; Raparelli et al. 2020; Wiviott et al. 2019) Although cardiovascular events were not assessed in this study, the effects of dapagliflozin on several cardiovascular risk factors (e.g., HbAlc, body weight, BP) observed were similar to those demonstrated in the DECLARE-TIMI 58 trial. In addition, change in lipid profile by dapagliflozin was similar to that reported in a pooled analysis of clinical trials.(Jabbour et al. 2018) While the slightly elevated LDL-C level may be of concern, a study showed that dapagliflozin increases concentration of the less atherogenic large buoyant LDL-C, while the potent atherogenic small dense LDL-C remained suppressed.(Hayashi et al. 2017) Future study with long-term follow-up would be expected to assess the cardiovascular outcomes with dapagliflozin treatment in the Taiwanese population.

Our study provided clinical effects of dapagliflozin on glycemic, weight, BP control at 6 months using a large representative cohort of patients withT2DM in Taiwan. Besides, comprehensive analyses were performed to identify subgroups and baseline predictors for better dapagliflozin response. However, there are several limitations in this study. First, due to a lack of comparison arm in prospective fashion, the reported effect size might not reflect the true differences. In addition, limited by the retrospective observational study design, partial loss of follow-up, missing data, and other confounding factors were inevitable. Due to the relatively low percentage of patients with prior CAD (7.8\%), cerebrovascular disease $(1.7 \%)$, and heart failure $(2.6 \%)$, we were unable to further analyzed the outcomes stratified by primary and secondary prevention groups. Besides, the safety data was not prospectively collected. However, we have recorded the reasons for discontinuation due to intolerance. In addition, disease duration and renal function (eGFR) were not recorded, so we were unable to confirm their association with dapagliflozin response in HbA1c, as observed in other studies.(Brown et al. 2017; Han et al. 2018; Wilding et al. 2017)

\section{Conclusions}

The LEAD study presented the effectiveness of dapagliflozin observed from the highest number of Chinese patients with T2DM in a real-world setting to date. Add-on therapy showed better glycemic control than switch therapy. The initiation of dapagliflozin was associated with significant improvements in glycemic control, body weight, and BP in Taiwanese patients, which is comparable to that in RCTs and other observational studies.

\section{Acknowledgements}


382

383

384

385

386

387

388

389

390

391

392

393

394

395

396

397

398

399

400

401

402

403

404

405

406

407

408

409

410

411

412

413

414

415

416

417

418

419

420

421

422

423

424

425

426

427

428

429

The study was supported by AstraZeneca Taiwan, Ltd. Writing and editorial assistance in the preparation of this manuscript was provided by Chian-Yi Liu at EMD Asia Scientific Communication (Taiwan branch) Co., Ltd, funded by AstraZeneca Taiwan, Ltd. The content of this article was expressed according to the opinions of authors and the manuscript submission for publication was completed with the final approval of all authors.

\section{References}

American Diabetes Association. 2019. 9. Pharmacologic Approaches to Glycemic Treatment: Standards of Medical Care in Diabetes-2019. Diabetes Care 42:S90-S102. 10.2337/dc19-S009

Bailey CJ, Gross JL, Hennicken D, Iqbal N, Mansfield TA, and List JF. 2013. Dapagliflozin addon to metformin in type 2 diabetes inadequately controlled with metformin: a randomized, double-blind, placebo-controlled 102-week trial. BMC Med 11:43. 10.1186/1741-7015-11-43

Bailey CJ, Morales Villegas EC, Woo V, Tang W, Ptaszynska A, and List JF. 2015. Efficacy and safety of dapagliflozin monotherapy in people with Type 2 diabetes: a randomized double-blind placebo-controlled 102-week trial. Diabet Med 32:531-541. $10.1111 / \mathrm{dme} .12624$

Bolinder J, Ljunggren O, Kullberg J, Johansson L, Wilding J, Langkilde AM, Sugg J, and Parikh S. 2012. Effects of dapagliflozin on body weight, total fat mass, and regional adipose tissue distribution in patients with type 2 diabetes mellitus with inadequate glycemic control on metformin. J Clin Endocrinol Metab 97:1020-1031. 10.1210/jc.2011-2260

Brown RE, Gupta N, and Aronson R. 2017. Effect of Dapagliflozin on Glycemic Control, Weight, and Blood Pressure in Patients with Type 2 Diabetes Attending a Specialist Endocrinology Practice in Canada: A Retrospective Cohort Analysis. Diabetes Technol Ther 19:685-691. 10.1089/dia.2017.0134

Cai X, Yang W, Gao X, Chen Y, Zhou L, Zhang S, Han X, and Ji L. 2018. The Association Between the Dosage of SGLT2 Inhibitor and Weight Reduction in Type 2 Diabetes Patients: A Meta-Analysis. 26:70-80. 10.1002/oby.22066

Cavender MA, Norhammar A, Birkeland KI, Jorgensen ME, Wilding JP, Khunti K, Fu AZ, Bodegard J, Blak BT, Wittbrodt E, Thuresson M, Fenici P, Hammar N, Kosiborod M, Investigators C-R, and Study G. 2018. SGLT-2 Inhibitors and Cardiovascular Risk: An Analysis of CVD-REAL. J Am Coll Cardiol 71:2497-2506. 10.1016/j.jacc.2018.01.085

Cho NH, Shaw JE, Karuranga S, Huang Y, da Rocha Fernandes JD, Ohlrogge AW, and Malanda B. 2018. IDF Diabetes Atlas: Global estimates of diabetes prevalence for 2017 and projections for 2045. Diabetes Res Clin Pract 138:271-281. 10.1016/j.diabres.2018.02.023

Del Prato S, Nauck M, Duran-Garcia S, Maffei L, Rohwedder K, Theuerkauf A, and Parikh S. 2015. Long-term glycaemic response and tolerability of dapagliflozin versus a sulphonylurea as add-on therapy to metformin in patients with type 2 diabetes: 4-year data. Diabetes Obes Metab 17:581-590. 10.1111/dom.12459

Diabetes Association Of The Republic Of China Taiwan. 2019. Executive summary of the DAROC clinical practice guidelines for diabetes care- 2018. J Formos Med Assoc. 10.1016/j.jfma.2019.02.016

Fadini GP, Zatti G, Baldi I, Bottigliengo D, Consoli A, Giaccari A, Sesti G, Avogaro A, and network D-TD. 2018. Use and effectiveness of dapagliflozin in routine clinical practice: An Italian multicentre retrospective study. Diabetes Obes Metab 20:1781-1786. 10.1111/dom.13280 
430

431

432

433

434

435

436

437

438

439

440

441

442

443

444

445

446

447

448

449

450

451

452

453

454

455

456

457

458

459

460

461

462

463

464

465

466

467

468

469

470

471

472

473

474

475

476

477

478

479

480

Garber AJ, Abrahamson MJ, Barzilay JI, Blonde L, Bloomgarden ZT, Bush MA, Dagogo-Jack S, DeFronzo RA, Einhorn D, Fonseca VA, Garber JR, Garvey WT, Grunberger G, Handelsman Y, Hirsch IB, Jellinger PS, McGill JB, Mechanick JI, Rosenblit PD, and Umpierrez GE. 2018. Consensus Statement by the American Association of Clinical Endocrinologists and American College of Endocrinology on the Comprehensive Type 2 Diabetes Management Algorithm - 2018 Executive Summary. Endocr Pract 24:91-120. 10.4158/CS-2017-0153

Garrison LP, Jr., Neumann PJ, Erickson P, Marshall D, and Mullins CD. 2007. Using real-world data for coverage and payment decisions: the ISPOR Real-World Data Task Force report. Value Health 10:326-335. 10.1111/j.1524-4733.2007.00186.x

Gomes MB, Rathmann W, Charbonnel B, Khunti K, Kosiborod M, Nicolucci A, Pocock SJ, Shestakova MV, Shimomura I, Tang F, Watada H, Chen H, Cid-Ruzafa J, Fenici P, Hammar N, Surmont F, Ji L, and investigators D. 2019. Treatment of type 2 diabetes mellitus worldwide: Baseline patient characteristics in the global DISCOVER study. Diabetes Res Clin Pract 151:20-32. 10.1016/j.diabres.2019.03.024

Han E, Kim A, Lee SJ, Kim JY, Kim JH, Lee WJ, and Lee BW. 2018. Characteristics of Dapagliflozin Responders: A Longitudinal, Prospective, Nationwide Dapagliflozin Surveillance Study in Korea. Diabetes Ther. 10.1007/s13300-018-0470-9

Hasan FM, Alsahli M, and Gerich JE. 2014. SGLT2 inhibitors in the treatment of type 2 diabetes. Diabetes Res Clin Pract 104:297-322. 10.1016/j.diabres.2014.02.014

Hayashi T, Fukui T, Nakanishi N, Yamamoto S, Tomoyasu M, Osamura A, Ohara M, Yamamoto T, Ito $Y$, and Hirano T. 2017. Dapagliflozin decreases small dense low-density lipoprotein-cholesterol and increases high-density lipoprotein 2-cholesterol in patients with type 2 diabetes: comparison with sitagliptin. Cardiovasc Diabetol 16:8. 10.1186/s12933-016-0491-5

Hong AR, Koo BK, Kim SW, Yi KH, and Moon MK. 2019. Efficacy and Safety of SodiumGlucose Cotransporter-2 Inhibitors in Korean Patients with Type 2 Diabetes Mellitus in Real-World Clinical Practice. Diabetes Metab J. 10.4093/dmj.2018.0134

Huang $\mathrm{CH}$, Huang YY, and Hsu BR. 2018a. A short-term follow-up of glycemic and body weight changes in diabetic patients who replaced dipeptidyl peptidase-4 inhibitors with the sodium-glucose cotransporter 2. Ann Saudi Med 38:420-426. 10.5144/02564947.2018.420

Huang H, Bell KF, Gani R, Tugwell CW, Eudicone JM, and Krukas-Hampel MR. 2018b. A retrospective real-world study of dapagliflozin versus other oral antidiabetic drugs added to metformin in patients with type 2 diabetes. Am J Manag Care 24:S132-S137.

International Diabetes Federation. 2019. IDF Diabetes Atlas, 9th edn. Brussels, Belgium: 2019. Available at: https://www. diabetesatlas.org.

Jabbour S, Seufert J, Scheen A, Bailey CJ, Karup C, and Langkilde AM. 2018. Dapagliflozin in patients with type 2 diabetes mellitus: A pooled analysis of safety data from phase IIb/III clinical trials. Diabetes Obes Metab 20:620-628. 10.1111/dom.13124

Jiang YD, Chang CH, Tai TY, Chen JF, and Chuang LM. 2012. Incidence and prevalence rates of diabetes mellitus in Taiwan: analysis of the 2000-2009 Nationwide Health Insurance database. J Formos Med Assoc 111:599-604. 10.1016/j.jfma.2012.09.014

Leiter LA, Cefalu WT, de Bruin TW, Xu J, Parikh S, Johnsson E, and Gause-Nilsson I. 2016. Long-term maintenance of efficacy of dapagliflozin in patients with type 2 diabetes mellitus and cardiovascular disease. Diabetes Obes Metab 18:766-774. 10.1111/dom. 12666

Lim LL, and Chan JCN. 2019. Sodium-glucose co-transporter-2 inhibitors: know the patient and the drugs. Hong Kong Med J 25:268-270. 10.12809/hkmj195089

Lim LL, Tan AT, Moses K, Rajadhyaksha V, and Chan SP. 2017. Place of sodium-glucose cotransporter-2 inhibitors in East Asian subjects with type 2 diabetes mellitus: Insights

Peerj reviewing PDF | (2020:02:45716:2:0:CHECK 12 Aug 2020) 
481

482

483

484

485

486

487

488

489

490

491

492

493

494

495

496

497

498

499

500

501

502

503

504

505

506

507

508

509

510

511

512

513

514

515

516

517

518

519

520

521

522

523

524

525

526

527

528

529

530

into the management of Asian phenotype. J Diabetes Complications 31:494-503. 10.1016/j.jdiacomp.2016.10.008

Ma RC, and Chan JC. 2013. Type 2 diabetes in East Asians: similarities and differences with populations in Europe and the United States. Ann N Y Acad Sci 1281:64-91.

10.1111/nyas.12098

Matthaei S, Bowering K, Rohwedder K, Sugg J, Parikh S, Johnsson E, and Study G. 2015. Durability and tolerability of dapagliflozin over 52 weeks as add-on to metformin and sulphonylurea in type 2 diabetes. Diabetes Obes Metab 17:1075-1084. 10.1111/dom.12543

McGuire H, Longson D, Adler A, Farmer A, Lewin I, and Guideline Development G. 2016. Management of type 2 diabetes in adults: summary of updated NICE guidance. BMJ 353:i1575. 10.1136/bmj.i1575

Mirabelli M, Chiefari E, Caroleo P, Vero R, Brunetti FS, Corigliano DM, Arcidiacono B, Foti DP, Puccio L, and Brunetti A. 2019. Long-Term Effectiveness and Safety of SGLT-2 Inhibitors in an Italian Cohort of Patients with Type 2 Diabetes Mellitus. J Diabetes Res 2019:3971060. 10.1155/2019/3971060

Molugulu N, Yee LS, Ye YT, Khee TC, Nie LZ, Yee NJ, Yee TK, Liang TC, and Kesharwani P. 2017. Systematic review of metformin monotherapy and dual therapy with sodium glucose co-transporter 2 inhibitor (SGLT-2) in treatment of type 2 diabetes mellitus. Diabetes Res Clin Pract 132:157-168. 10.1016/j.diabres.2017.07.025

Nanditha A, Ma RC, Ramachandran A, Snehalatha C, Chan JC, Chia KS, Shaw JE, and Zimmet PZ. 2016. Diabetes in Asia and the Pacific: Implications for the Global Epidemic. Diabetes Care 39:472-485. 10.2337/dc15-1536

Nauck MA, Del Prato S, Duran-Garcia S, Rohwedder K, Langkilde AM, Sugg J, and Parikh SJ. 2014. Durability of glycaemic efficacy over 2 years with dapagliflozin versus glipizide as add-on therapies in patients whose type 2 diabetes mellitus is inadequately controlled with metformin. Diabetes Obes Metab 16:1111-1120. 10.1111/dom.12327

Norhammar A, Bodegard J, Nystrom T, Thuresson M, Nathanson D, and Eriksson JW. 2019. Dapagliflozin and cardiovascular mortality and disease outcomes in a population with type 2 diabetes similar to that of the DECLARE-TIMI 58 trial: A nationwide observational study. Diabetes Obes Metab 21:1136-1145. 10.1111/dom.13627

Raparelli V, Elharram M, Moura CS, Abrahamowicz M, Bernatsky S, Behlouli H, and Pilote L. 2020. Sex Differences in Cardiovascular Effectiveness of Newer Glucose-Lowering Drugs Added to Metformin in Type 2 Diabetes Mellitus. J Am Heart Assoc 9:e012940. 10.1161/JAHA.119.012940

Scheerer MF, Rist R, Proske O, Meng A, and Kostev K. 2016. Changes in HbA1c, body weight, and systolic blood pressure in type 2 diabetes patients initiating dapagliflozin therapy: a primary care database study. Diabetes Metab Syndr Obes 9:337-345. 10.2147/DMSO.S116243

Scholtes RA, van Baar MJB, Lytvyn Y, Bjornstad P, Nieuwdorp M, Cherney DZI, and van Raalte DH. 2019. Sodium glucose cotransporter (SGLT)-2 inhibitors: Do we need them for glucose-lowering, for cardiorenal protection or both? Diabetes Obes Metab 21 Suppl 2:24-33. 10.1111/dom.13692

Scorsone A, Saura G, Fleres M, Spano L, Aiello V, Brancato D, Di Noto A, Provenzano F, and Provenzano V. 2018. Efficacy and Renal Safety of Dapagliflozin in Patients with Type 2 Diabetes Mellitus Also Receiving Metformin: A Real-Life Experience. J Diabetes Res 2018:8501418. 10.1155/2018/8501418

Sun YN, Zhou Y, Chen X, Che WS, and Leung SW. 2014. The efficacy of dapagliflozin combined with hypoglycaemic drugs in treating type 2 diabetes mellitus: meta-analysis of randomised controlled trials. BMJ Open 4:e004619. 10.1136/bmjopen-2013-004619 
531

532

533

534

535

536

537

538

539

540

541

542

543

544

545

546

547

548

549
Tobita H, Sato S, Miyake T, Ishihara S, and Kinoshita Y. 2017. Effects of Dapagliflozin on Body Composition and Liver Tests in Patients with Nonalcoholic Steatohepatitis Associated with Type 2 Diabetes Mellitus: A Prospective, Open-label, Uncontrolled Study. Curr Ther Res Clin Exp 87:13-19. 10.1016/j.curtheres.2017.07.002

Viswanathan V, and Singh KP. 2019. Use of Dapagliflozin in the Management of Type 2 Diabetes Mellitus: A Real-World Evidence Study in Indian Patients (FOREFRONT). Diabetes Technol Ther 21:415-422. 10.1089/dia.2019.0052

Wilding J, Bailey C, Rigney U, Blak B, Kok M, and Emmas C. 2017. Dapagliflozin therapy for type 2 diabetes in primary care: Changes in $\mathrm{HbA1c}$, weight and blood pressure over 2 years follow-up. Prim Care Diabetes 11:437-444. 10.1016/j.pcd.2017.04.004

Wiviott SD, Raz I, Bonaca MP, Mosenzon O, Kato ET, Cahn A, Silverman MG, Zelniker TA, Kuder JF, Murphy SA, Bhatt DL, Leiter LA, McGuire DK, Wilding JPH, Ruff CT, GauseNilsson IAM, Fredriksson M, Johansson PA, Langkilde AM, Sabatine MS, and Investigators D-T. 2019. Dapagliflozin and Cardiovascular Outcomes in Type 2 Diabetes. N Engl J Med 380:347-357. 10.1056/NEJMoa1812389

Zhang M, Zhang L, Wu B, Song H, An Z, and Li S. 2014. Dapagliflozin treatment for type 2 diabetes: a systematic review and meta-analysis of randomized controlled trials. Diabetes Metab Res Rev 30:204-221. 10.1002/dmrr.2479 
Figure 1

Changes in $\mathrm{HbAlC}$ at 3 and 6 months (A) by baseline $\mathrm{HbAlC}$ and (B) by manner of dapagliflozin initiation. 

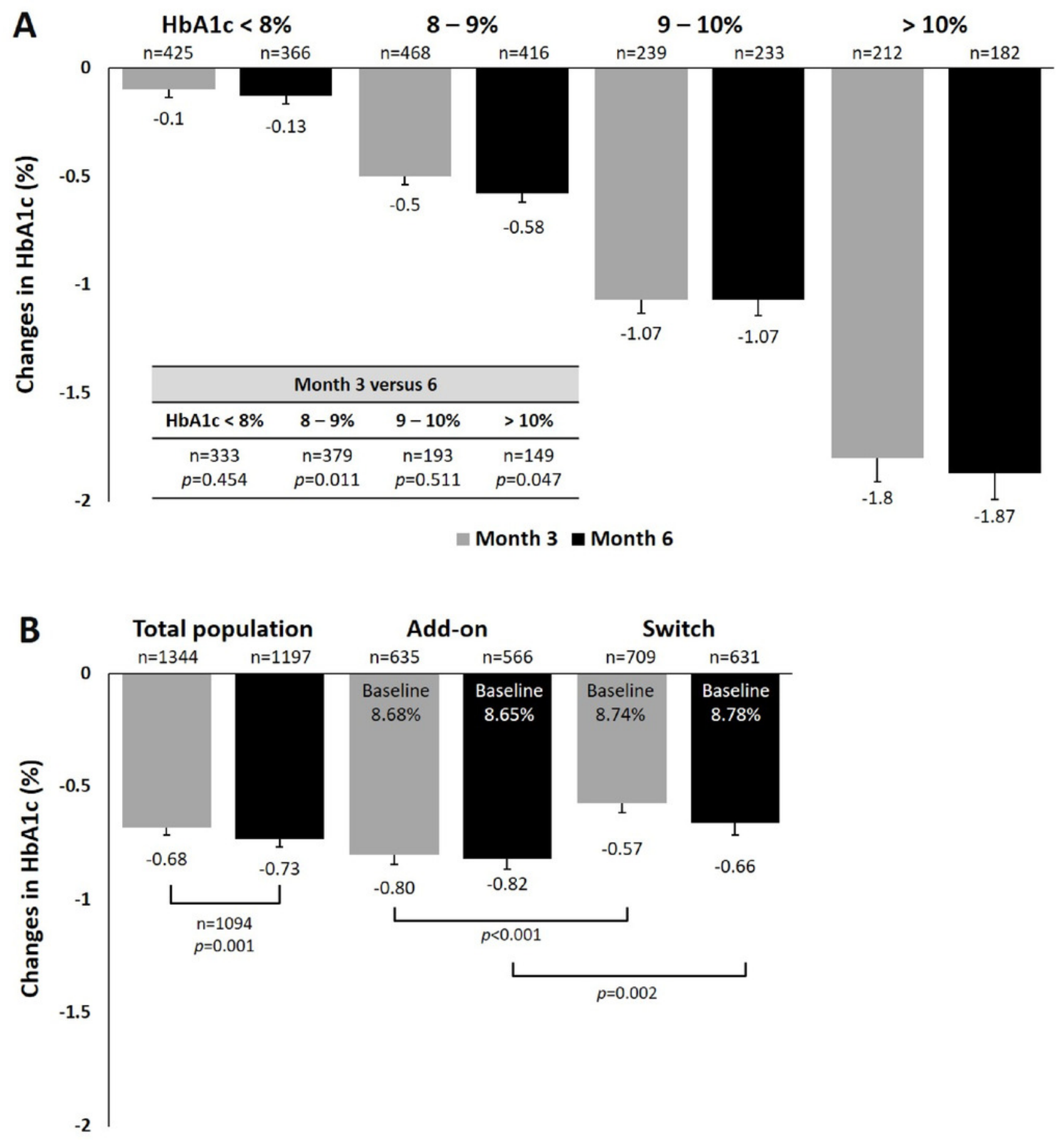

Month 3 Month 6 
Figure 2

Changes in body weight at 3 and 6 months by baseline BMI.

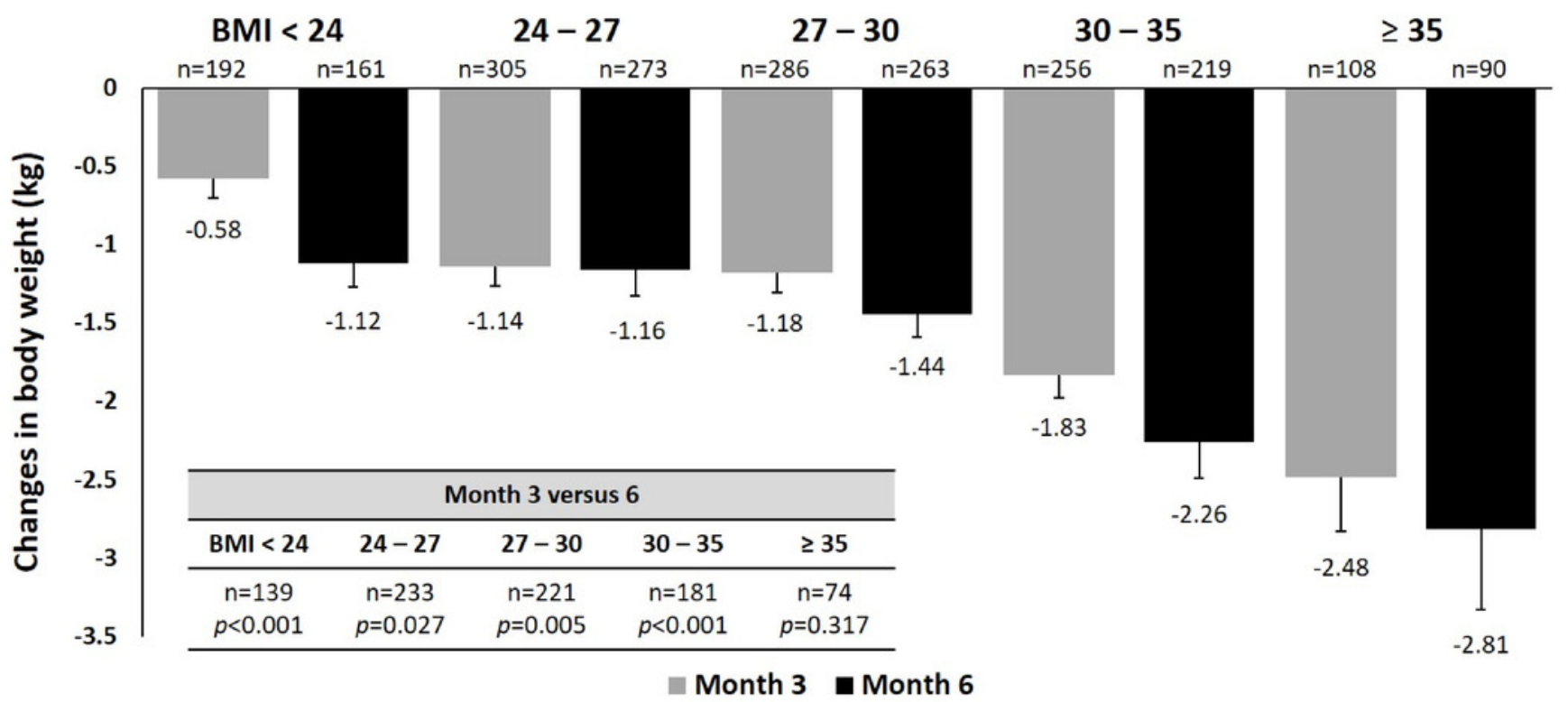


Figure 3

Scatter plots for the relationship between changes in $\mathrm{HbAlc}$ and body weight at Month 6 among (A) total, (B) add-on, and (C) switch populations.
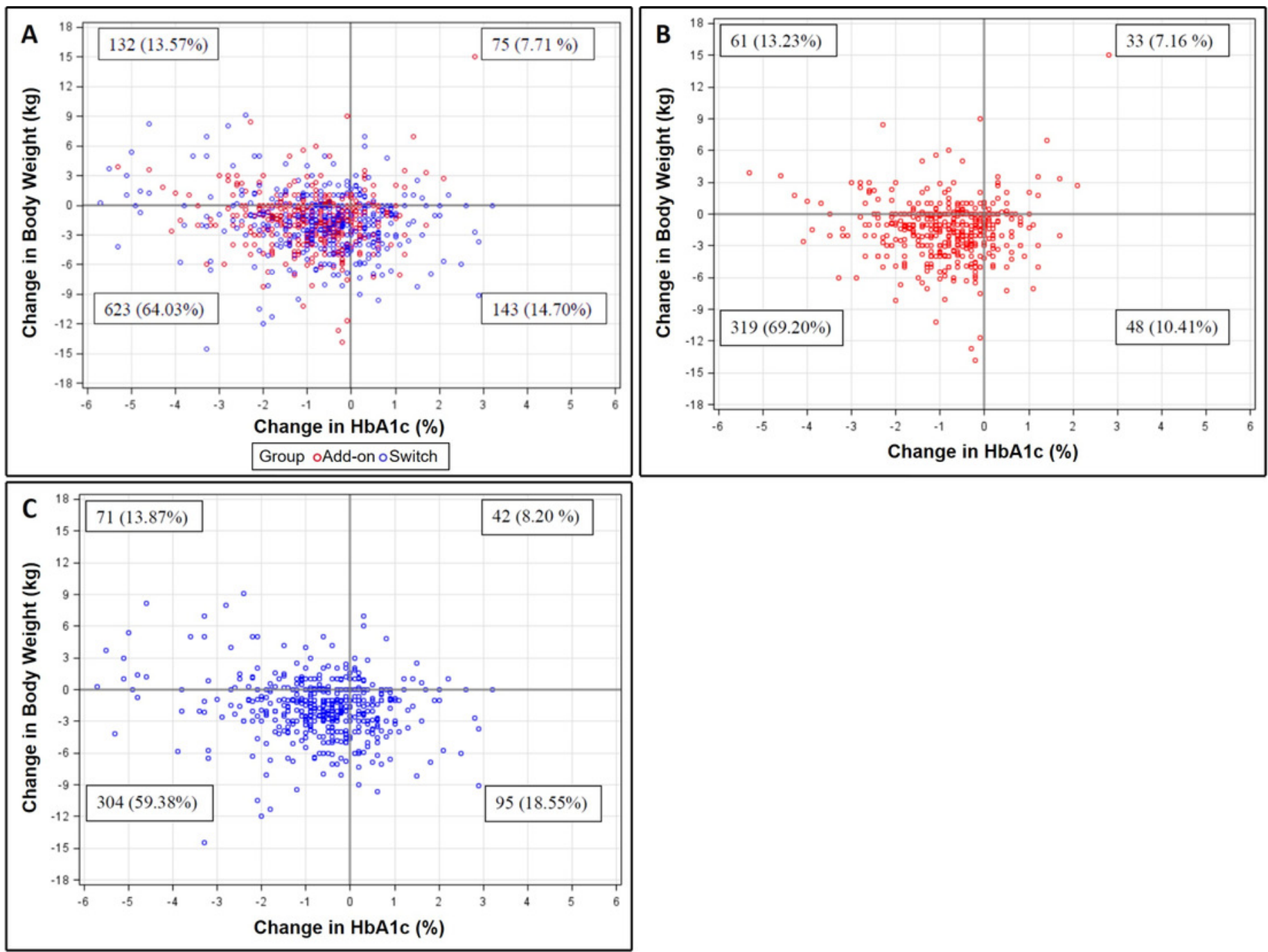
Table $\mathbf{1}$ (on next page)

Baseline characteristics in 1960 patients. 
1 Table 1. Baseline characteristics in 1960 patients.

\begin{tabular}{|c|c|}
\hline Age (years) & $57.8 \pm 11.5$ \\
\hline Sex (male) & $1020(52 \%)$ \\
\hline Weight (kg) & $75.2 \pm 15.8$ \\
\hline BMI $\left(\mathrm{kg} / \mathrm{m}^{2}\right)$ & $28.3 \pm 4.9$ \\
\hline \multicolumn{2}{|l|}{ BMI categories } \\
\hline$<24$ & $17.6 \%$ \\
\hline$\geq 24-<27$ & $26.0 \%$ \\
\hline$\geq 27-<30$ & $24.9 \%$ \\
\hline$\geq 30-<35$ & $22.3 \%$ \\
\hline$\geq 35$ & $9.2 \%$ \\
\hline HbA1c (\%) & $8.8 \pm 1.4$ \\
\hline \multicolumn{2}{|l|}{ HbA1c distribution } \\
\hline$<7 \%$ & $5.8 \%$ \\
\hline$\geq 7 \%-<8 \%$ & $25.1 \%$ \\
\hline$\geq 8 \%-<9 \%$ & $31.4 \%$ \\
\hline$>9 \%-\leq 10 \%$ & $19.6 \%$ \\
\hline$>10 \%$ & $18.1 \%$ \\
\hline $\mathrm{FPG}(\mathrm{mg} / \mathrm{dL})$ & $173.0 \pm 53.9$ \\
\hline $\mathrm{SBP} / \mathrm{DBP}(\mathrm{mmHg})$ & $136.6 \pm 17.6 / 77.8 \pm 11.3$ \\
\hline$\underline{\mathrm{DBP}}(\mathrm{mmHg})$ & $\underline{77.8 \pm 11.3}$ \\
\hline Total cholesterol (mg/dL) & $164.3 \pm 36.0$ \\
\hline Triglycerides (mg/dL) & $171.2 \pm 133.6$ \\
\hline LDL-C (mg/dL) & $89.8 \pm 27.8$ \\
\hline $\mathrm{HDL}-\mathrm{C}(\mathrm{mg} / \mathrm{dL})$ & $44.8 \pm 13.5$ \\
\hline \multicolumn{2}{|l|}{ Medical history } \\
\hline Hypertension & $60.5 \%$ \\
\hline CAD & $7.8 \%$ \\
\hline Cerebrovascular disease & $1.7 \%$ \\
\hline PAD & $7.4 \%$ \\
\hline Heart failure & $2.6 \%$ \\
\hline Nephropathy & $34.6 \%$ \\
\hline Retinopathy & $10.7 \%$ \\
\hline Neuropathy & $14.1 \%$ \\
\hline Antihyperglycemic therapy & \\
\hline
\end{tabular}




\begin{tabular}{|c|c|}
\hline Metformin & $92.4 \%$ \\
\hline Sulfonylurea & $70.7 \%$ \\
\hline DPP4 inhibitor & $53.5 \%$ \\
\hline Meglitinide & $3.0 \%$ \\
\hline$\alpha$-glucosidase inhibitor & $16.7 \%$ \\
\hline Thiazolidinedione & $26.6 \%$ \\
\hline GLP-1 RA & $3.0 \%$ \\
\hline Insulin & $29.5 \%$ \\
\hline \multicolumn{2}{|c|}{ Number of antihyperglycemic drug prior to dap } \\
\hline Monotherapy & $11.9 \%$ \\
\hline Dual therapy & $38.2 \%$ \\
\hline Triple therapy & $36.2 \%$ \\
\hline$>3$ drugs combination & $13.8 \%$ \\
\hline \multicolumn{2}{|c|}{ Manner of dapagliflozin initiation } \\
\hline Add-on & $905(46.2 \%)$ \\
\hline Switch & $1055(53.8 \%)$ \\
\hline \multicolumn{2}{|l|}{ Dosage of dapagliflozin } \\
\hline Dapagliflozin 5 mg & $413(21.1 \%)$ \\
\hline Dapagliflozin 10 mg & $1547(78.9 \%)$ \\
\hline Antihypertensive therapy & $61.7 \%$ \\
\hline ACEI or ARB & $49.6 \%$ \\
\hline$\beta$-blocker & $21.1 \%$ \\
\hline Calcium channel blocker & $30.9 \%$ \\
\hline Diuretic & $11.3 \%$ \\
\hline Others & $3.0 \%$ \\
\hline Lipid-lowering therapy & $74.5 \%$ \\
\hline Statin & $68.6 \%$ \\
\hline Fibrate & $9.7 \%$ \\
\hline Niacin & $2.3 \%$ \\
\hline Ezetimibe & $9.6 \%$ \\
\hline \multicolumn{2}{|l|}{ Antithrombotic therapy } \\
\hline Antiplatelet & $22.5 \%$ \\
\hline Anticoagulant & $2.1 \%$ \\
\hline
\end{tabular}

2 Values are in $\mathrm{n}(\%)$, mean $\pm \mathrm{SD}$, or percent of total population. The following parameters had

3 patients ( $n$ ) with missing data: Weight, $n=192 ; B M I, n=206 ; \mathrm{HbA} 1 \mathrm{c}, \mathrm{n}=119$; FPG, $n=380$; 
4 SBP/DBP, $n=210 ;$ Total cholesterol, $n=910 ;$ Triglycerides, $n=772 ; H D L-C, n=1052 ; L D L-C, n=$ 5538.

$6 \mathrm{ACEI}$, angiotensin-converting-enzyme inhibitors; ARB, angiotensin II receptor blockers; BMI, 7 body mass index; CAD, coronary artery disease; DBP, diastolic blood pressure; DPP4, dipeptidyl 8 peptidase-4; FPG, fasting plasma glucose; GLP-1 RA, glucagon-like peptide-1 receptor agonists; $9 \mathrm{HbA1C}$, glycated hemoglobin; HDL-C, high-density lipoprotein cholesterol; LDL-C, low-density 10 lipoprotein cholesterol; PAD, peripheral artery disease; SBP, systolic blood pressure. 


\section{PeerJ}


Table 2 (on next page)

Changes in clinical and laboratory outcomes from baseline to 3 and 6 months. 
Table 2. Changes in clinical and laboratory outcomes from baseline to 3 and 6 months.

\begin{tabular}{|c|c|c|c|c|c|c|c|c|c|}
\hline \multirow[t]{2}{*}{ Parameter } & \multicolumn{3}{|c|}{ Month 3} & \multicolumn{3}{|c|}{ Month 6} & \multicolumn{3}{|c|}{ Month 3 versus Month 6} \\
\hline & $n$ & Change & $p$ value & $n$ & Change & $p$ value & $\mathbf{n}$ & Change & $p$ value \\
\hline $\mathrm{HbA} 1 \mathrm{c}, \%$ & $\begin{array}{l}134 \\
4\end{array}$ & $\begin{array}{l}-0.68(-0.74,- \\
0.62)\end{array}$ & $\begin{array}{l}<0.00 \theta \\
1\end{array}$ & $\begin{array}{l}119 \\
7\end{array}$ & $\begin{array}{l}-0.73(-0.80,- \\
0.67)\end{array}$ & $<0.0001$ & $\begin{array}{l}109 \\
4\end{array}$ & $\begin{array}{l}-0.07(-0.11 \\
0.02)\end{array}$ & 0.001 \\
\hline $\mathrm{BMI}, \mathrm{kg} / \mathrm{m}^{2}$ & $\begin{array}{l}114 \\
7\end{array}$ & $\begin{array}{l}-0.50(-0.56,- \\
0.45)\end{array}$ & $\begin{array}{l}<0.000 \\
1\end{array}$ & $\begin{array}{l}100 \\
6\end{array}$ & $\begin{array}{l}-0.60(-0.67,- \\
0.54)\end{array}$ & $<0.0001$ & 878 & $\begin{array}{l}-0.14(-0.18,- \\
0.09)\end{array}$ & $\begin{array}{l}<0.00 \theta \\
1\end{array}$ \\
\hline Body weight, kg & $\begin{array}{l}115 \\
6\end{array}$ & $\begin{array}{l}-1.34(-1.48,- \\
1.20)\end{array}$ & $\begin{array}{l}<0.000 \\
1\end{array}$ & $\begin{array}{l}101 \\
5\end{array}$ & $\begin{array}{l}-1.61(-1.79,- \\
1.42)\end{array}$ & $<0.0001$ & 884 & $\begin{array}{l}-0.35(-0.48,- \\
0.23)\end{array}$ & $\begin{array}{l}<0.00 \theta \\
1\end{array}$ \\
\hline $\mathrm{FPG}, \mathrm{mg} / \mathrm{dL}$ & $\begin{array}{l}110 \\
8\end{array}$ & $\begin{array}{l}-26.6(-29.6,- \\
23.7)\end{array}$ & $\begin{array}{l}<0.00 \theta \\
1\end{array}$ & 963 & $\begin{array}{l}-28.3(-31.6,- \\
25.1)\end{array}$ & $<0.0001$ & 919 & $-2.0(-4.7,0.6)$ & 0.014 \\
\hline $\mathrm{SBP}, \mathrm{mmHg}$ & $\begin{array}{l}114 \\
3\end{array}$ & $-3.9(-4.8,-3.0)$ & $\begin{array}{l}<0.000 \\
1\end{array}$ & 997 & $-3.6(-4.6,-2.6)$ & $<0.0001$ & 872 & $0.2(-0.8,1.3)$ & $\begin{array}{l}0.85 \underline{3} z \\
7\end{array}$ \\
\hline $\mathrm{DBP}, \mathrm{mmHg}$ & $\begin{array}{l}114 \\
3\end{array}$ & $-1.7(-2.3,-1.1)$ & $\begin{array}{l}<0.000 \\
1\end{array}$ & 997 & $-1.4(-2.0,-0.7)$ & $\leq 0.00 \underline{1} \theta$ & 872 & $0.4(-0.3,1.1)$ & $0.161 \theta$ \\
\hline $\begin{array}{l}\text { Total cholesterol, } \\
\mathrm{mg} / \mathrm{dL}\end{array}$ & 485 & $3.6(0.6,6.6)$ & $\frac{\leq 0.00 \theta}{1}$ & 446 & $2.9(-0.2,6.0)$ & 0.0063 & 433 & $-3.2(-6.4,0.1)$ & 0.5856 \\
\hline Triglycerides, mg/dL & 587 & $-6.9(-22.8,9.0)$ & $\begin{array}{l}<0 . \theta 00 \\
1\end{array}$ & 542 & $\begin{array}{l}-16.6(-27.0,- \\
6.2)\end{array}$ & $<0.0001$ & 501 & $-12.5(-31.7,6.7)$ & 0.0864 \\
\hline LDL-C, mg/dL & 797 & $0.58(-1.4,2.5)$ & $\begin{array}{l}0.00 \underline{9} 8 \\
5\end{array}$ & 730 & $1.1(-0.9,3.1)$ & $0.014 \underline{35}$ & 635 & $-2.5(-4.6,-0.5)$ & 0.1800 \\
\hline $\mathrm{HDL}-\mathrm{C}, \mathrm{mg} / \mathrm{dL}$ & 400 & $1.1(0.2,2.1)$ & $\begin{array}{l}<0.000 \\
1\end{array}$ & 369 & $2.4(1.2,3.7)$ & $<0.0001$ & 371 & $1.6(0.8,2.3)$ & $\begin{array}{l}<0.00 \theta \\
1\end{array}$ \\
\hline
\end{tabular}

Data are presented as mean (95\% confidence interval).

$3 \mathrm{BMI}$, body mass index; DBP, diastolic blood pressure; FPG, fasting plasma glucose; HbA1c, glycated hemoglobin; HDL-C, high-density

4 lipoprotein cholesterol; LDL-C, low-density lipoprotein cholesterol; SBP, systolic blood pressure. 


\section{Table 3(on next page)}

Baseline factors associated with dapagliflozin response in $\mathrm{HbAlc}$ at Month 6 examined by logistic regression analysis. 
1 Table 3. Baseline factors associated with dapagliflozin response in $\mathrm{HbA1C}$ at Month 6 examined

2 by logistic regression analysis.

\begin{tabular}{|c|c|c|c|}
\hline Parameter & OR & $95 \% \mathrm{Cl}$ & $p$ value \\
\hline \multicolumn{4}{|l|}{ HbA1c-Univariate } \\
\hline Age (years) & 0.994 & $(0.984,1.0105)$ & $0.27 \underline{769}$ \\
\hline Gender, female & $1.20 z$ & $(0.943,1.53 z)$ & $0.138 \theta$ \\
\hline Weight (kg) & $1.00 z$ & $(0.994,1.01 \theta)$ & 0.59438 \\
\hline BMI $\left(\mathrm{kg} / \mathrm{m}^{2}\right)$ & 1.013 & $(0.9 \underline{9} 86,1.041)$ & 0.3573 \\
\hline HbA1c (\%) & 2.1219 & $(1.8 \underline{766}, 2.4 \underline{106})$ & $<0.00 \theta 1$ \\
\hline $\mathrm{FPG}(\mathrm{mg} / \mathrm{dL})$ & 1.010 & $(1.007,1.013)$ & $<0.0001$ \\
\hline SBP (mmHg) & $1.00 \theta$ & $(0.99 z, 1.0107)$ & 0.9551 \\
\hline Add-on dapagliflozin & 1.310 & $(1.0 \underline{3} z 8,1.67 \theta)$ & 0.0293 \\
\hline \multicolumn{4}{|c|}{ Number of antihyperglycemic drug } \\
\hline Monotherapy & 0.764 & $(0.473,1.2435)$ & $0.27 \underline{3} z 6$ \\
\hline Dual therapy & $0.66 z$ & $(0.4 \underline{5} 49,0.9 \underline{875})$ & 0.03769 \\
\hline Triple therapy & $0.72 \theta$ & $(0.4 \underline{9} 88,1.06 z)$ & 0.09877 \\
\hline \multicolumn{4}{|c|}{ Antihyperglycemic therapy } \\
\hline Insulin & 1.5876 & $(1.200,2.07 \theta)$ & 0.0011 \\
\hline \multicolumn{4}{|l|}{ HbA1c-Multivariate } \\
\hline HbA1c (\%) & 2.10099 & $(1.7 \underline{9} 86,2.4768)$ & $<0.0001$ \\
\hline $\mathrm{FPG}(\mathrm{mg} / \mathrm{dL})$ & $1.00 z$ & $\begin{array}{l}(\underline{1.000 .998,} \\
1.0105)\end{array}$ & $0.34 \underline{9} 86$ \\
\hline Add-on dapagliflozin & $1 . \underline{60596}$ & $(1.191,2.14 \underline{37})$ & $0.00 \underline{217}$ \\
\hline \multicolumn{4}{|c|}{ Number of antihyperglycemic drug } \\
\hline Monotherapy & 1.401 & $(0.784,2.504)$ & $0.255 \theta$ \\
\hline Dual therapy & 0.941 & $(0.581,1.523)$ & 0.8043 \\
\hline Triple therapy & 0.953 & $(0.589,1.541)$ & $0.84 \underline{4} 38$ \\
\hline \multicolumn{4}{|c|}{ Antihyperglycemic therapy } \\
\hline Insulin & $0.83 z$ & $(0.589,1.1876)$ & $0.298 \theta$ \\
\hline
\end{tabular}

3 Note: Responders and non-responders were determined by using the median of change in 4 HbA1c (-0.60\%) as the cutoff.

5 Abbreviations: $\mathrm{BMI}$, body mass index; $\mathrm{Cl}$, confidence interval; $\mathrm{FPG}$, fasting plasma glucose;

$6 \mathrm{HbA1c}$, glycated hemoglobin; OR, odds ratio; SBP, systolic blood pressure. 